\title{
GENERALIZED CONTINUED FRACTION EXPANSIONS WITH CONSTANT PARTIAL DENOMINATORS
}

\section{TOPI TÖRMÄ}

(September 20, 2018)

\begin{abstract}
We study generalized continued fraction expansions of the form

$$
\frac{a_{1}}{N}+\frac{a_{2}}{N}+\frac{a_{3}}{N}+\cdots,
$$

where $N$ is a fixed positive integer and the partial numerators $a_{i}$ are positive integers for all $i$. We call these expansions $\mathrm{dn}_{N}$ expansions and show that every positive real number has infinitely many $\mathrm{dn}_{N}$ expansions for each $N$. In particular we study the $\mathrm{dn}_{N}$ expansions of rational numbers and quadratic irrationals. Finally we show that every positive real number has for each $N$ a $\mathrm{dn}_{N}$ expansion with bounded partial numerators.
\end{abstract}

Keywords and phrases: Generalized continued fractions Mathematics Subject Classification (2010): $11 J^{\text {r70. }}$

\section{Introduction}

In [1] Anselm and Weintraub introduced a generalization of simple continued fractions, the $\mathrm{cf}_{N}$ expansion

$$
a_{0}+\frac{N}{a_{1}}+\frac{N}{a_{2}}+\frac{N}{a_{3}}+\cdots
$$

where $N$ is a fixed positive integer, $a_{0}$ is a non-negative integer and $a_{i}$ is a positive integer for every $i$. They showed that every positive real number has infinitely many $\mathrm{cf}_{N}$ expansions for all $N>1$ and studied the properties of these expansions for rational numbers and quadratic irrationals. In particular, they focused on the so-called best $\mathrm{cf}_{N}$ expansion, where the partial denominators $a_{i}$ are chosen as large as possible and which is unique for each real number.

(c) XXXX Australian Mathematical Society 0263-6115/XX $\$ A 2.00+0.00$ 
In this paper we flip the roles of the partial numerators and denominators of the $\mathrm{cf}_{N}$ expansions and study generalized continued fraction expansions of the form

$$
\frac{a_{1}}{N}+\frac{a_{2}}{N}+\frac{a_{3}}{N}+\cdots
$$

where $N$ is a fixed positive integer and $a_{i}$ are positive integers. We shall call these continued fractions $\operatorname{dn}_{N}$ expansions and denote them by $\left\langle a_{1}, a_{2}, \ldots\right\rangle_{N}$. While a general study of the $\mathrm{dn}_{N}$ expansions of real numbers doesn't seem to have been done, continued fractions of form (1) have been studied quite a lot. For example, Ramanujan presented many such continued fractions in his notebooks $([2],[3])$. Among them were

$$
1=\frac{x+N}{N}+\frac{(x+N)^{2}-N^{2}}{N}+\frac{(x+2 N)^{2}-N^{2}}{N}+\frac{(x+3 N)^{2}-N^{2}}{N}+\cdots
$$

where $x \neq-k N$ for all positive integers $k$,

$$
1+2 N^{2} \sum_{k=1}^{\infty} \frac{(-1)^{k}}{(N+k)^{2}}=\frac{1}{N}+\frac{1^{2}}{N}+\frac{1 \cdot 2}{N}+\frac{2^{2}}{N}+\frac{2 \cdot 3}{N}+\frac{3^{2}}{N}+\cdots
$$

and perhaps most famously Ramanujan's AGM continued fraction

$$
\mathcal{R}_{N}(a, b)=\frac{a}{N}+\overline{b^{2}}+\frac{(2 a)^{2}}{N}+\frac{(3 b)^{2}}{N}+\frac{(4 a)^{2}}{N}+\frac{(5 b)^{2}}{N}+\cdots
$$

that satisfies the remarkable equation

$$
\mathcal{R}_{N}\left(\frac{a+b}{2}, \sqrt{a b}\right)=\frac{\mathcal{R}_{N}(a, b)+\mathcal{R}_{N}(b, a)}{2}
$$

connecting the arithmetic and geometric mean of numbers $a$ and $b([4])$. Some examples of well-known $\mathrm{dn}_{N}$ expansions for real numbers are Lord Brouncker's $\mathrm{dn}_{2}$ expansion

$$
\pi=\frac{8}{2}+\frac{2}{2}+\frac{3^{2}}{2}+\frac{5^{2}}{2}+\cdots
$$

(see $[6]$ ), the $\mathrm{dn}_{1}$ expansion

$$
\ln 2=\mathcal{R}_{1}(1,1)=\frac{1}{1}+\frac{1^{2}}{1}+\frac{2^{2}}{1}+\frac{3^{2}}{1}+\cdots
$$

(see [4]), and the $\mathrm{dn}_{1}$ expansion

$$
\zeta(2)-1=\frac{\pi^{2}}{6}-1=\frac{1}{1}+\frac{1^{2}}{1}+\frac{1 \cdot 2}{1}+\frac{2^{2}}{1}+\frac{2 \cdot 3}{1}+\frac{3^{2}}{1}+\cdots
$$

derived from (2). 
We will begin with some preliminaries in Section 2, followed by the $\mathrm{dn}_{N}$ algorithm in Section 3. We will show that every positive real number has infinitely many $\operatorname{dn}_{N}$ expansions for every $N$ and define a special $\operatorname{dn}_{N}$ expansion called the least $\mathrm{dn}_{N}$ expansion. In Sections 4 and 5 we will examine the $\operatorname{dn}_{N}$ expansions of positive rational numbers and positive real quadratic irrationals, respectively. We will prove that for any rational number there exist infinitely many finite, periodic and aperiodic $\mathrm{dn}_{N}$ expansions, and that for any quadratic irrational number there exist infinitely many periodic and aperiodic $\mathrm{dn}_{N}$ expansions. Special attention is paid to the least $\mathrm{dn}_{N}$ expansion of these numbers. In Section 6 we will show that every positive real number has a $\mathrm{dn}_{N}$ expansion with bounded partial numerators.

In this paper, we denote the set of positive integers with $\mathbb{Z}_{+}$and the set of non-negative integers with $\mathbb{N}$.

\section{On continued fractions}

We begin with some preliminaries on (generalized) continued fractions

$$
b_{0}+\frac{a_{1}}{b_{1}+\frac{a_{2}}{b_{2}+\cdots}}=b_{0}+\stackrel{\infty}{K}_{n=1}^{\infty} \frac{a_{n}}{b_{n}}=b_{0}+\frac{a_{1}}{b_{1}}+\frac{a_{2}}{b_{2}}+\cdots,
$$

where the partial numerators $a_{n}$ and the partial denominators $b_{n}$ are positive integers for all $n \in \mathbb{Z}_{+}$and $b_{0} \in \mathbb{Z}$. If the limit of the $n$ :th convergent

$$
\frac{A_{n}}{B_{n}}=b_{0}+\frac{a_{1}}{b_{1}}+\frac{a_{2}}{b_{2}}+\cdots+\frac{a_{n}}{b_{n}}
$$

at infinity exists, it is called the value of the continued fraction. The numerators $A_{n}$ and denominators $B_{n}$ of the convergents can be obtained from the recurrence relations

$$
\left\{\begin{array}{l}
A_{n+2}=b_{n+2} A_{n+1}+a_{n+2} A_{n} \\
B_{n+2}=b_{n+2} B_{n+1}+a_{n+2} B_{n}
\end{array}\right.
$$

with initial values $A_{0}=b_{0}, B_{0}=1, A_{1}=b_{0} b_{1}+a_{1}$ and $B_{1}=b_{1}$. These relations imply the formula

$$
\frac{A_{n+1}}{B_{n+1}}-\frac{A_{n}}{B_{n}}=\frac{(-1)^{n} a_{1} \ldots a_{n+1}}{B_{n} B_{n+1}},
$$

valid for all $n \in \mathbb{N}$. If continued fraction (3) converges to $\tau \in \mathbb{R}$, then

$$
\tau=b_{0}+\sum_{k=0}^{\infty} \frac{(-1)^{k} a_{1} \ldots a_{k+1}}{B_{k} B_{k+1}},
$$


as shown for example in [5]. Using recurrence relations (4) and the standard error estimates of alternating series we get

$$
\frac{b_{n+2} a_{1} \ldots a_{n+1}}{B_{n} B_{n+2}}<\left|\tau-\frac{A_{n}}{B_{n}}\right|<\frac{a_{1} \ldots a_{n+1}}{B_{n} B_{n+1}} .
$$

We can also determine the sign of $\tau-A_{n} / B_{n}$ since equations (4) and (5) imply

$$
\frac{A_{0}}{B_{0}}<\frac{A_{2}}{B_{2}}<\ldots<\frac{A_{2 k}}{B_{2 k}}<\tau<\frac{A_{2 l+1}}{B_{2 l+1}}<\ldots<\frac{A_{3}}{B_{3}}<\frac{A_{1}}{B_{1}}
$$

for all $k, l \in \mathbb{N}$.

As the partial coefficients $a_{n}$ and $b_{n}$ of continued fraction (3) are positive integers for all $n \in \mathbb{Z}_{+}$, the following theorem gives us a convergence criterion.

Theorem 2.1. [The Seidel-Stern Theorem] Let $a_{n}$ and $b_{n}$ be positive real numbers for all $n$. Then the continued fraction $\mathrm{K}_{n=1}^{\infty} \frac{a_{n}}{b_{n}}$ converges if and only if the Stern-Stolz series

$$
\sum_{n=1}^{\infty} b_{n} \prod_{k=1}^{n} a_{k}^{(-1)^{n-k+1}}
$$

diverges to $\infty$. 2 .

Proof. See [7], Chapter III, Theorem 3 and the subsequent Remark

Corollary 2.2. Let $a_{n}$ and $b_{n}$ be positive integers for all $n$. If the sequence $\left(a_{n}\right)$ has a bounded subsequence, then the continued fraction $\mathrm{K}_{n=1}^{\infty} \frac{a_{n}}{b_{n}}$ converges.

Proof. Let us assume that $\left(a_{n}\right)$ has a bounded subsequence $\left(a_{k_{i}}\right)$ such that $a_{k_{i}} \leq M$ for all $i \in \mathbb{Z}_{+}$and some $M \in \mathbb{Z}_{+}$. Without loss of generality, we may also assume that $k_{i+1} \geq k_{i}+2$. By denoting

$$
S_{n}=\prod_{k=1}^{n} a_{k}^{(-1)^{n-k+1}}
$$

the Stern-Stolz series of the continued fraction $\mathrm{K}_{n=1}^{\infty} \frac{a_{n}}{b_{n}}$ can be written as $\sum_{n=1}^{\infty} b_{n} S_{n}$, where $S_{1}=1 / a_{1}$ and $S_{n+1}=1 /\left(S_{n} a_{n+1}\right)$. Now either $S_{k_{i}} \geq 1$ or $S_{k_{i}}<1$ and $S_{k_{i}+1}=1 /\left(S_{k_{i}} a_{k_{i}+1}\right)>1 / M$ so

$$
\sum_{n=1}^{\infty} b_{n} S_{n} \geq \sum_{i=1}^{\infty}\left(S_{k_{i}}+S_{k_{i}+1}\right) \geq \sum_{i=1}^{\infty} \frac{1}{M} \rightarrow \infty .
$$

Hence the Stern-Stolz series of $\mathrm{K}_{n=1}^{\infty} \frac{a_{n}}{b_{n}}$ diverges to infinity and by Theorem 2.1 the continued fraction $\mathrm{K}_{n=1}^{\infty} \frac{a_{n}}{b_{n}}$ converges. 
We say that the (infinite) expansion $\left\langle a_{1}, a_{2}, \ldots\right\rangle_{N}$ is (eventually) periodic if there exist positive integers $k$ and $m$ such that $a_{i}=a_{i+k}$ for every $i \geq m$. Then we denote

$$
\left\langle a_{1}, a_{2}, \ldots\right\rangle_{N}=\left\langle a_{1}, \ldots, a_{m-1}, \overline{a_{m}, \ldots, a_{m+k-1}}\right\rangle_{N} .
$$

Every periodic $\mathrm{dn}_{N}$ expansion converges by Corollary 2.2 since the partial numerators of periodic continued fractions are bounded. It is easy to see that every periodic $\mathrm{dn}_{N}$ expansion represents a rational number or a quadratic irrational.

Finally, we recall some useful results from the theory of simple continued fractions

$$
c_{0}+\frac{1}{c_{1}}+\frac{1}{c_{2}}+\ldots=\left[c_{0} ; c_{1}, c_{2}, \ldots\right]
$$

which are a special case of continued fractions (3) with $a_{n}=1$ and $b_{n}=c_{n}$ for all $n$. We denote the convergents of the simple continued fraction expansion by $C_{n} / D_{n}$. As is well known, the simple continued fraction expansion of a real number $\tau$ is finite if and only if $\tau$ is rational and periodic if and only if $\tau$ is a quadratic irrational. Especially,

$$
\sqrt{d}=\left[c_{0} ; \overline{c_{1}, \ldots, c_{k-1}, 2 c_{0}}\right],
$$

where $d$ is a positive non-square integer, $c_{0}=\lfloor\sqrt{d}\rfloor$ and $c_{i}=c_{k-i}$ for all $1 \leq i \leq k-1$ (see [8]).

For simple continued fractions error estimates (6) take the form

$$
\frac{1}{\left(d_{n+1}+2\right) D_{n}^{2}}<\frac{d_{n+2}}{D_{n} D_{n+2}}<\left|\tau-\frac{C_{n}}{D_{n}}\right|<\frac{1}{D_{n} D_{n+1}}<\frac{1}{d_{n+1} D_{n}^{2}},
$$

which suggests the convergents $C_{n} / D_{n}$ are good approximants for $\tau$. In a way they are the only very good approximants as the following Theorem shows:

TheOREM 2.3. If $\tau$ is a real number, $p \in \mathbb{Z}$ and $q \in \mathbb{N}$ are coprime and

$$
\left|\tau-\frac{p}{q}\right|<\frac{1}{2 q^{2}}
$$

then $p / q$ is a convergent of the simple continued fraction expansion of $\tau$.

For the proof, see for example Lemma 2.33 in [5].

\section{The $\mathrm{dn}_{N}$ expansion}

Through the rest of this paper, $N$ is a fixed positive integer and $\tau_{0}$ is an arbitrary positive real number unless stated otherwise.

We now present the $\mathrm{dn}_{N}$ algorithm for obtaining a $\mathrm{dn}_{N}$ expansion for $\tau_{0}$ : 
1) Let $i=1$.

2) Choose a positive integer $a_{i}$ such that $a_{i} / \tau_{i-1} \geq N$.

3) Let $\tau_{i}=\frac{a_{i}}{\tau_{i-1}}-N$. If $\tau_{i}=0$, terminate. Otherwise let $i=i+1$ and go to step 2 .

As the only criterion for choosing each $a_{i}$ is to keep $\tau_{i}$ non-negative, we can obtain uncountably many $\mathrm{dn}_{N}$ expansions for $\tau_{0}$. However, we would like our continued fraction to converge to the number $\tau_{0}$. Therefore the partial numerators $a_{i}$ should be chosen so that the series

$$
\sum_{n=1}^{\infty} \prod_{k=1}^{n} a_{k}^{(-1)^{n-k+1}}
$$

diverges to infinity, which in the case of $\mathrm{dn}_{N}$ expansions implies the divergence of the Stern-Stolz series (8).

LEMmA 3.1. If the $\mathrm{dn}_{N}$ expansion obtained for $\tau_{0}$ by the $d n_{N}$ algorithm converges, then it converges to $\tau_{0}$.

Proof. By induction,

$$
\tau_{0}=\frac{A_{n}+A_{n-1} \tau_{n}}{B_{n}+B_{n-1} \tau_{n}} .
$$

Then

$$
\begin{aligned}
\left|\tau_{0}-\frac{A_{n}}{B_{n}}\right| & =\left|\frac{A_{n}+A_{n-1} \tau_{n}}{B_{n}+B_{n-1} \tau_{n}}-\frac{A_{n}}{B_{n}}\right| \\
& =\left|\frac{A_{n} B_{n}+A_{n-1} B_{n} \tau_{n}-A_{n} B_{n}-A_{n} B_{n-1} \tau_{n}}{B_{n}\left(B_{n}+B_{n-1} \tau_{n}\right)}\right| \\
& =\frac{\tau_{n} \prod_{i=1}^{n} a_{i}}{B_{n}\left(B_{n}+B_{n-1} \tau_{n}\right)}<\frac{\prod_{i=1}^{n} a_{i}}{B_{n} B_{n-1}} .
\end{aligned}
$$

Since the continued fraction converges, we have

$$
\lim _{n \rightarrow \infty} \frac{\prod_{i=1}^{n} a_{i}}{B_{n} B_{n-1}}=0
$$

by (5), and hence $\lim _{n \rightarrow \infty} A_{n} / B_{n}=\tau_{0}$.

In the multitude of possibilities for choosing the partial numerators there is a natural method for making the choices uniquely, and that is by choosing each $a_{i}$ to be as small as possible. Since the smallest positive integer $a_{i}$ such that $\tau_{i}=a_{i} / \tau_{i-1}-N \geq 0$ is $\left\lceil N \tau_{i-1}\right\rceil$, we give the following definition: 
Definition 1. The $\operatorname{dn}_{N}$ expansion of $\tau_{0}$ obtained from the $\mathrm{dn}_{N}$ algorithm by choosing $a_{i}=\left\lceil N \tau_{i-1}\right\rceil$ for every $i$ is the least $\operatorname{dn}_{N}$ expansion of $\tau_{0}$.

TheOrem 3.2. The least $\mathrm{dn}_{N}$ expansion of $\tau_{0}$ converges.

Proof. If the least $\mathrm{dn}_{N}$ expansion of $\tau_{0}$ is finite, we interpret it as converging. Let the least $\mathrm{dn}_{N}$ expansion $\left\langle a_{1}, a_{2}, \ldots\right\rangle_{N}$ of $\tau_{0}$ be infinite. If $\tau_{i} \geq 1$, then

$$
0<\tau_{i+1}=\frac{\left\lceil N \tau_{i}\right\rceil}{\tau_{i}}-N<\frac{N \tau_{i}+1}{\tau_{i}}-N=\frac{1}{\tau_{i}} \leq 1
$$

so there are infinitely many $i$ such that $\tau_{i} \leq 1$. Since $\tau_{i} \leq 1$ implies that $a_{i+1}=\left\lceil N \tau_{i}\right\rceil \leq N$, there are infinitely many $i$ such that $a_{i} \leq N$. Therefore the sequence $\left(a_{i}\right)$ has a bounded subsequence, so by Corollary 2.2 the continued fraction $\left\langle a_{1}, a_{2}, \ldots\right\rangle_{N}$ converges and by Lemma 3.1 it converges to $\tau_{0}$.

We have now established that every positive real number has at least one converging $\mathrm{dn}_{N}$ expansion. In fact, there are uncountably many such expansions since we may choose $a_{i}=\left\lceil N \tau_{i-1}\right\rceil+1$ instead of $a_{i}=\left\lceil N \tau_{i-1}\right\rceil$ and still get a converging $\mathrm{dn}_{N}$ expansion. From this point forward, when we talk about a $\mathrm{dn}_{N}$ expansion $\left\langle a_{1}, a_{2}, \ldots\right\rangle_{N}$ of a positive real number $\tau_{0}$, we indicate that the expansion converges to $\tau_{0}$, that is $\tau_{0}=\left\langle a_{1}, a_{2}, \ldots\right\rangle_{N}$.

EXAMPLE 1. Here are some least $\mathrm{dn}_{N}$ expansions of different numbers.

\begin{tabular}{|c|c|c|}
\hline$\tau_{0}$ & $N$ & least $\operatorname{dn}_{N}$ expansion of $\tau_{0}$ \\
\hline $5 / 17$ & 1 & $\langle 1,3,1,3\rangle_{1}$ \\
& 10 & $\langle 3,2\rangle_{10}$ \\
\hline$\sqrt{2}$ & 1 & $\langle 2,1\rangle_{1}$ \\
& 2 & $\langle 3,1,13,1,21,1,24,1,27,1,136,1,140,1,7849, \ldots\rangle_{2}$ \\
& 7 & $\langle 10, \overline{1,50}\rangle_{7}$ \\
\hline$\pi$ & 1 & $\langle 4,1,3,1,7,1,37,1,71,1,449,1,657,1,991, \ldots\rangle_{1}$ \\
& 2 & $\langle 7,1,5,1,17,1,20,1,108,1,204,1,239,1,326, \ldots\rangle_{2}$ \\
\hline$e$ & 1 & $\langle 3,1,9,1,24,1,65,1,67,1,335,1,881,1,1152, \ldots\rangle_{1}$ \\
& 7 & $\langle 20,3,10,2,23,2,5,6,4,5,9,2,4,2,22, \ldots\rangle_{7}$ \\
\hline
\end{tabular}

\section{Rational numbers}

Throughout this section $\tau_{0}=p / q$ is a positive rational number with $p, q \in \mathbb{Z}_{+}$. 
THEOREM 4.1. Let $\left(k_{j}\right)$ be a sequence of positive integers such that $k_{j} q>$ $N$ for all $j$. Then

$$
\begin{aligned}
\frac{p}{q} & =\left\langle k_{1} p, k_{1}^{2} q^{2}-N^{2}, k_{1}(p+N q), k_{2} p, k_{2}^{2} q^{2}-N^{2}, k_{2}(p+N q), \ldots\right\rangle_{N} \\
& \left.=\underset{j=1}{\left\langle k_{j} p, k_{j}^{2} q^{2}-N^{2}, k_{j}(p+N q)\right.}\right\rangle_{N} .
\end{aligned}
$$

Proof. With the choices

$$
\begin{cases}a_{3 j-2} & =k_{j} p \\ a_{3 j-1} & =k_{j}^{2} q^{2}-N^{2} \\ a_{3 j} & =k_{j}(p+N q)\end{cases}
$$

for all $j \in \mathbb{Z}_{+}$we get inductively from the $\operatorname{dn}_{N}$ algorithm that

$$
\begin{gathered}
\tau_{3 j-2}=\frac{k_{j} p}{p / q}-N=k_{j} q-N>0, \\
\tau_{3 j-1}=\frac{k_{j}^{2} q^{2}-N^{2}}{k_{j} q-N}-N=k_{j} q
\end{gathered}
$$

and

$$
\tau_{3 j}=\frac{k_{j}(p+N q)}{k_{j} q}-N=\frac{p}{q}=\tau_{0} .
$$

Hence we obtain the $\mathrm{dn}_{N}$ expansion

$$
\underset{j=1}{\infty}\left\langle\overline{k_{j} p, k_{j}^{2} q^{2}-N^{2}, k_{j}(p+N q)}\right\rangle_{N} .
$$

Using the same notation as in the proof of Corollary 2.2, if $S_{3 j-3} \leq 1$, then since $k_{j} q \geq N+1$, we have

$$
S_{3 j}=\frac{a_{3 j-1}}{a_{3 j-2} a_{3 j} S_{3 j-3}}>\frac{k_{j}^{2} q^{2}-N^{2}}{k_{j}^{2} p(p+N q)} \geq \frac{q^{2}(2 N+1)}{p(p+N q)(N+1)^{2}} .
$$

Therefore the sequence $\left(S_{3 j}\right)$ is bounded below by a positive constant and the Stern-Stolz series of continued fraction (10) diverges to infinity. Then the convergence of continued fraction (10) to $p / q$ follows from Theorem 2.1 and Lemma 3.1.

COROLlary 4.2. The rational number $p / q$ has infinitely many periodic $\mathrm{dn}_{N}$ expansions and uncountably many aperiodic $\mathrm{dn}_{N}$ expansions.

Proof. By Theorem 4.1 the rational number $p / q$ has the $\mathrm{dn}_{N}$ expansion (10) for any sequence of positive integers $\left(k_{j}\right)$ that satisfies $k_{j} q>N$ for all $j$. If $\left(k_{j}\right)$ is periodic of period length $m$, then the $\mathrm{dn}_{N}$ expansion

$$
\frac{p}{q}=\underset{j=1}{\infty}\left\langle\overline{k_{j} p, k_{j}^{2} q^{2}-N^{2}, k_{j}(p+N q)}\right\rangle_{N}
$$


is periodic of period length $3 m$ at most. As there are infinitely many periodic sequences $\left(k_{j}\right)$, it follows that there are infinitely many periodic $\mathrm{dn}_{N}$ expansions for $p / q$.

On the other hand, if we choose the sequence $\left(k_{j}\right)$ to be such that it has a strictly increasing subsequence, then the $\mathrm{dn}_{N}$ exapansion (10) is aperiodic because it contains arbitrarily large partial numerators. As there are uncountably many such sequences $\left(k_{j}\right)$, there are uncountably many aperiodic $\mathrm{dn}_{N}$ expansions for $p / q$.

Example 2. Let $\tau_{0}=22 / 7$ and $N=13$. It is then that Theorem 4.1 gives the $\mathrm{dn}_{13}$ expansion

$$
\frac{22}{7}=\underset{j=1}{\infty}\left\langle\overline{22 k_{j}, 49 k_{j}^{2}-169,127 k_{j}}\right\rangle_{13},
$$

where the sequence $\left(k_{j}\right)$ satisfies $k_{j} \geq 2$ for all $j$. Using different sequences $\left(k_{j}\right)$ we get the following $\mathrm{dn}_{13}$ expansions:

\begin{tabular}{|c|c|}
\hline$\left(k_{j}\right)$ & $\mathrm{dn}_{13}$ expansion of $22 / 7$ \\
\hline$k_{j}=2$ for all $j$ & $\langle\overline{44,27,254}\rangle_{13}$ \\
\hline$k_{2 i-1}=2, k_{2 i}=3$ & $\langle\overline{44,27,254,66,272,381}\rangle_{13}$ \\
\hline$k_{j}=j+1$ for all $j$ & $\langle 44,27,254,66,272,381,88,615, \ldots\rangle_{13}$ \\
\hline
\end{tabular}

In Example 1 both of the least $\mathrm{dn}_{N}$ expansions calculated for $5 / 17$ were finite. It turns out this is the case for every least $\mathrm{dn}_{N}$ expansion of a positive rational number.

TheOREM 4.3. The least $\operatorname{dn}_{N}$ expansion of $\tau_{0}=p / q$ is finite.

Proof. Let us denote $P_{0}=p, Q_{0}=q$ and $S_{0}=P_{0}+Q_{0}$. By the division algorithm there exist unique $q_{1}, r_{1} \in \mathbb{N}$ such that $N P_{0}=q_{1} Q_{0}+r_{1}$, where $0<r_{1} \leq Q_{0}$. Then $\left\lceil N P_{0} / Q_{0}\right\rceil=q_{1}+1$. Using the $\operatorname{dn}_{N}$ algorithm we have

$$
\tau_{1}=\frac{\left\lceil N P_{0} / Q_{0}\right\rceil}{P_{0} / Q_{0}}-N=\frac{Q_{0}\left(q_{1}+1\right)-N P_{0}}{P_{0}}=\frac{Q_{0}-r_{1}}{P_{0}} .
$$

If $r_{1}=Q_{0}$, then $\tau_{1}=0$ and the algorithm terminates. If $0<r_{1}<Q_{0}$, we put $P_{1}=Q_{0}-r_{1}$ and $Q_{1}=P_{0}$. Note that now

$$
S_{0}=P_{0}+Q_{0}>P_{0}+Q_{0}-r_{1}=P_{1}+Q_{1}=S_{1} .
$$

Suppose we have reached $\tau_{i}=P_{i} / Q_{i}, P_{i}, Q_{i} \in \mathbb{Z}_{+}$and $S_{i}=P_{i}+Q_{i}$. By the division algorithm there exist unique $q_{i+1}, r_{i+1} \in \mathbb{N}$ such that

$$
N P_{i}=q_{i+1} Q_{i}+r_{i+1},
$$

where $0<r_{i+1} \leq Q_{i}$. Then $\left\lceil N P_{i} / Q_{i}\right\rceil=q_{i+1}+1$ and

$$
\tau_{i+1}=\frac{\left\lceil N P_{i} / Q_{i}\right\rceil}{P_{i} / Q_{i}}-N=\frac{Q_{i}\left(q_{i+1}+1\right)-N P_{i}}{P_{i}}=\frac{Q_{i}-r_{i+1}}{P_{i}} .
$$


If $r_{i+1}=Q_{i}$, then $\tau_{i+1}=0$ and the algorithm terminates. If $0<r_{i+1}<Q_{i}$, we put $P_{i+1}=Q_{i}-r_{i+1}$ and $Q_{i+1}=P_{i}$. Then

$$
S_{i}=P_{i}+Q_{i}>P_{i}+Q_{i}-r_{i+1}=P_{i+1}+Q_{i+1}=S_{i+1} .
$$

Because the sequence $\left(S_{i}\right)$ is a strictly decreasing sequence of positive integers and $Q_{i}>0$, it follows there must exist an $n \in \mathbb{Z}_{+}$such that $P_{n}=0$. Then $\tau_{n}=0$ and the algorithm terminates. Thus the least $\mathrm{dn}_{N}$ expansion of $\tau_{0}$ is finite.

We get infinitely many finite $\operatorname{dn}_{N}$ expansions for $p / q$ by choosing the first finitely many $a_{i}$ as we please and then making the least choice from there on.

\section{Quadratic irrationals}

Let us start by noting that because there are uncountably many infinite $\mathrm{dn}_{N}$ expansions for every positive real number but there exist only countably many periodic $\mathrm{dn}_{N}$ expansions, it follows that every positive quadratic irrational number has uncountably many aperiodic $\mathrm{dn}_{N}$ expansions.

Throughout this section $\tau_{0}$ is a positive real quadratic irrational. Now there exist $P, Q, d \in \mathbb{Z}$ such that $\tau_{0}=(\sqrt{d}+P) / Q, d \geq 2$ is not a perfect square and $Q \mid\left(d-P^{2}\right)$ (see for example [8], Lemma 10.5). Then we denote $Q^{\prime}=\left|\left(d-P^{2}\right) / Q\right|=|\sqrt{d}-P| \tau_{0}$.

Lemma 5.1. If $|P|<\sqrt{d}$ and $k \in \mathbb{Z}_{+}$is such that $k(\sqrt{d}-P)>N$, then

$$
\tau_{0}=\left\langle k Q^{\prime}, \overline{D-2 k P N-N^{2}, D}\right\rangle_{N},
$$

where $D=k^{2}\left(d-P^{2}\right)$.

Proof. Since $|P|<\sqrt{d}$ and $\tau_{0}$ is positive, it follows that $Q$ and $D$ are positive and $Q^{\prime}=\left(d-P^{2}\right) / Q$. If we choose $a_{1}=k Q^{\prime}$, we get from the $\operatorname{dn}_{N}$ algorithm

$$
\tau_{1}=\frac{k Q^{\prime}}{\tau_{0}}-N=\frac{k\left(d-P^{2}\right)}{\sqrt{d}+P}-N=k \sqrt{d}-(k P+N)>0 .
$$

As $k(\sqrt{d}+P)+N>0$, we may continue by choosing

$$
a_{2}=D-2 k P N-N^{2}=k^{2} d-(k P+N)^{2}=(k(\sqrt{d}+P)+N) \tau_{1}>0
$$

and get

$$
\tau_{2}=\frac{(k(\sqrt{d}+P)+N) \tau_{1}}{\tau_{1}}-N=k \sqrt{d}+k P>0
$$


Finally with $a_{3}=D$ we have

$$
\tau_{3}=\frac{k^{2}\left(d-P^{2}\right)}{k \sqrt{d}+k P}-N=k \sqrt{d}-(k P+N)=\tau_{1},
$$

and thus we get the periodic expansion $\tau_{0}=\left\langle k Q^{\prime}, \overline{D-2 k P N-N^{2}, D}\right\rangle_{N}$.

THEOREM 5.2. There exists a periodic $\mathrm{dn}_{N}$ expansion of the positive real quadratic irrational $\tau_{0}$.

Proof. We begin by constructing the desired $\mathrm{dn}_{N}$ expansion for $\tau_{0}$. Let us denote $P_{0}=P, Q_{0}=Q$ and $R_{0}=1$. Let $k_{1}$ be the smallest positive integer such that $k_{1}\left|\sqrt{d}-P_{0}\right|>N$ and $a_{1}=k_{1} Q^{\prime}$. Then we get from the $\operatorname{dn}_{N}$ algorithm

$$
\tau_{1}=\frac{a_{1}}{\tau_{0}}-N=\frac{k_{1}\left|\sqrt{d}-P_{0}\right| \tau_{0}}{\tau_{0}}-N=k_{1}\left|\sqrt{d}-P_{0}\right|-N>0 .
$$

Now we denote $\tau_{1}=R_{1} \sqrt{d}+P_{1}$, where

$$
\begin{cases}R_{1}=k_{1} \text { and } P_{1}=-k_{1} P_{0}-N, & \text { when } \sqrt{d}>P_{0}, \\ R_{1}=-k_{1} \text { and } P_{1}=k_{1} P_{0}-N, & \text { when } \sqrt{d}<P_{0} .\end{cases}
$$

If $\tau_{i}=R_{i} \sqrt{d}+P_{i}$, we choose $a_{i+1}=k_{i+1}\left|R_{i}^{2} d-P_{i}^{2}\right|$, where $k_{i+1}$ is the smallest positive integer such that $k_{i+1}\left|R_{i} \sqrt{d}-P_{i}\right|>N$ and get

$$
\tau_{i+1}=\frac{k_{i+1}\left|R_{i}^{2} d-P_{i}^{2}\right|}{R_{i} \sqrt{d}+P_{i}}-N=k_{i+1}\left|R_{i} \sqrt{d}-P_{i}\right|-N>0 .
$$

Then we denote $\tau_{i+1}=R_{i+1} \sqrt{d}+P_{i+1}$, where

$$
\begin{cases}R_{i+1}=k_{i+1} R_{i} \text { and } P_{i+1}=-k_{i+1} P_{i}-N, & \text { when } R_{i} \sqrt{d}>P_{i}, \\ R_{i+1}=-k_{i+1} R_{i} \text { and } P_{i+1}=k_{i+1} P_{i}-N, & \text { when } R_{i} \sqrt{d}<P_{i} .\end{cases}
$$

It remains to be shown that the $\operatorname{dn}_{N}$ expansion $\left\langle a_{1}, a_{2}, \ldots\right\rangle_{N}$ constructed above is periodic. Note that if we choose $k$ in Lemma 5.1 to be as small as possible, then the periodic $\mathrm{dn}_{N}$ expansion (11) is a special case of the $\mathrm{dn}_{N}$ expansion under study. Therefore it suffices to show that there exists a $j \geq 1$ such that $R_{j}$ is positive and

$$
\left|P_{j}\right|<\left|R_{j} \sqrt{d}\right|=R_{j} \sqrt{d}=\sqrt{R_{j}^{2} d}
$$

in which case Lemma 5.1 gives us the periodicity.

Suppose on the contrary that

$$
\left|P_{i}\right|>\left|R_{i} \sqrt{d}\right| \text { for all } i \geq 1 .
$$


Then $P_{i}$ is positive for all $i$ because $\tau_{i}=R_{i} \sqrt{d}+P_{i}$ is positive for all $i$. If $k_{i}=1$ for every large $i$, then $P_{i+1}=P_{i}-N$ and $R_{i+1}=-R_{i}$ for every large $i$. In this case the sequence $\left(P_{i}\right)$ is a strictly decreasing sequence of integers and so there exists a $j$ such that $P_{j}<0$, which we can't have. Hence there exist infinitely many $j$ such that $k_{j}>1$. This implies that the sequence $\left(\left|R_{i}\right|\right)$ is tending to infinity so there exists an $n$ such that $\left|R_{i} \sqrt{d}\right|>N$ for all $i \geq n$.

Let $m \geq n$ be such that $k_{m+1} \geq 2$. As $k_{m+1}$ is the least positive integer such that

$$
k_{m+1} P_{m}-k_{m+1} R_{m} \sqrt{d}=k_{m+1}\left|P_{m}-R_{m} \sqrt{d}\right|>N
$$

then

$$
\left(k_{m+1}-1\right) P_{m}-\left(k_{m+1}-1\right) R_{m} \sqrt{d}<N .
$$

Combining the above inequalities yields

$$
0<k_{m+1} P_{m}-k_{m+1} R_{m} \sqrt{d}-N<P_{m}-R_{m} \sqrt{d}<N,
$$

where the last inequality holds because $k_{m+1} \geq 2$. Since $P_{m}>\left|R_{m} \sqrt{d}\right|>N$ and $P_{m}-R_{m} \sqrt{d}<N$, then $R_{m}>0$ and

$$
P_{m+1}-R_{m+1} \sqrt{d}=k_{m+1} P_{m}-N+k_{m+1} R_{m} \sqrt{d}>3 N .
$$

Thus $k_{m+2}=1$ and by (13) we have

$$
P_{m+2}=P_{m+1}-N=k_{m+1} P_{m}-2 N<k_{m+1} R_{m} \sqrt{d}=R_{m+2} \sqrt{d},
$$

where $R_{m+2}=k_{m+1} R_{m}$ is positive. This is a contradiction with assumption (12). Thus there exists a $j \geq 1$ such that $R_{j}$ is positive and $\left|P_{j}\right|<R_{j} \sqrt{d}$ and by Lemma 5.1

$$
\tau_{0}=\left\langle a_{1}, a_{2}, \ldots, a_{j}, D / k_{j+1}, \overline{D-2 k_{j+1} P_{j} N-N^{2}, D}\right\rangle_{N},
$$

where $D=k_{j+1}^{2}\left(R_{j}^{2} d-P_{j}^{2}\right)$.

Since we may choose the first finitely many $a_{i}$ as we please and then continue as described in Lemma 5.1 and Theorem 5.2, every positive quadratic irrational has infinitely many different periodic $\mathrm{dn}_{N}$ expansions.

ExAmple 3. Let $\tau_{0}=(7+\sqrt{10}) / 13$ and $N=4$. Constructing the $\mathrm{dn}_{4}$ expansion described in Theorem 5.2 we get

$$
\begin{array}{lll}
k_{1}=2, & a_{1}=6, & \tau_{1}=10-2 \sqrt{10}, \\
k_{2}=1, & a_{2}=60, & \tau_{2}=6+2 \sqrt{10}, \\
k_{3}=13, & a_{3}=52, & \tau_{3}=26 \sqrt{10}-82, \\
k_{4}=1, & a_{4}=36, & \tau_{4}=26 \sqrt{10}+78, \\
k_{5}=1, & a_{5}=676, & \tau_{5}=26 \sqrt{10}-82=\tau_{3}
\end{array}
$$


and so

$$
\frac{7+\sqrt{10}}{13}=\langle 6,60,52, \overline{36,676}\rangle_{4} .
$$

We now turn our attention to the least $\mathrm{dn}_{N}$ expansions of positive real quadratic irrationals. In [1] it is conjectured that the best $\operatorname{cf}_{N}$ expansion of a positive quadratic irrational is not periodic for every $N$. It seems likely that this is the case for the least $\mathrm{dn}_{N}$ expansion as well. For example the $\mathrm{dn}_{1}$ expansion of $\sqrt{3}$ is

$$
\begin{aligned}
\sqrt{3}= & \langle 2,1,6,1,10,1,11,1,18,1,50,1,65,1,750,1,8399,1,11727,1,12855, \\
& 1,66368,1,281130,1,437015,1,482182,1,643701,1,743770,1, \\
& 2808107,1,11306550,1,12268089,1,24304646,1,98323268,1, \ldots\rangle_{1},
\end{aligned}
$$

where the partial numerators seem to alternate between 1 and a rapidly increasing sequence of positive integers.

However, there are some cases when we can find a periodic least $\mathrm{dn}_{N}$ expansion. Recall that if $|P|<\sqrt{d}$ and $k \in \mathbb{Z}_{+}$is such that $k(\sqrt{d}-P)>N$, then by Lemma 5.1

$$
\tau_{0}=\left\langle k Q^{\prime}, \overline{D-2 k P N-N^{2}, D}\right\rangle_{N},
$$

where $D=k^{2}\left(d-P^{2}\right)$.

Theorem 5.3. Let $|P|<\sqrt{d}$ and $k \in \mathbb{Z}_{+}$be such that $k(\sqrt{d}-P)>N$. Then expansion (14) is the least $\mathrm{dn}_{N}$ expansion of $\tau_{0}$ if and only if

$$
0<(\sqrt{d}-P)-\frac{N}{k}<\frac{1}{(\sqrt{d}+P) k^{2}} .
$$

Proof. As noted in the proof of 5.1, in this case the numbers $Q, Q^{\prime}$ and $D$ are positive integers. Expansion (14) is the least $\mathrm{dn}_{N}$ expansion if and only if $a_{i}=\left\lceil N \tau_{i-1}\right\rceil$ for every $i$. Since expansion (14) is periodic, it suffices to check that $k Q^{\prime}=\left\lceil N \tau_{0}\right\rceil, D-2 k P N-N^{2}=\left\lceil N \tau_{1}\right\rceil$ and $D=\left\lceil N \tau_{2}\right\rceil$. From the proof of 5.1 we have that $\tau_{1}=k \sqrt{d}-(k P+N)$ and $\tau_{2}=k \sqrt{d}+k P$. If $D=\left\lceil N \tau_{2}\right\rceil$ then

$$
k^{2}\left(d-P^{2}\right)=\lceil N k(\sqrt{d}+P)\rceil=N k(\sqrt{d}+P)+c,
$$

where $0<c<1$. Now

$$
k Q^{\prime}=N \frac{\sqrt{d}+P}{Q}+\frac{c}{k Q}=N \tau_{0}+\frac{c}{k Q},
$$

where $0<c / k Q<1$ and

$$
\begin{aligned}
D-2 k P N-N^{2} & =N k(\sqrt{d}+P)-2 k P N-N^{2}+c \\
& =N(k \sqrt{d}-(k P+N))+c=N \tau_{1}+c,
\end{aligned}
$$


so $k Q^{\prime}=\left\lceil N \tau_{0}\right\rceil$ and $D-2 k P N-N^{2}=\left\lceil N \tau_{1}\right\rceil$. It is therefore enough to study when $D=\left\lceil N \tau_{2}\right\rceil$.

By (16) $D=\left\lceil N \tau_{2}\right\rceil$ if and only if

$$
0<c=k^{2}\left(d-P^{2}\right)-N k(\sqrt{d}+P)=k(\sqrt{d}+P)(k(\sqrt{d}-P)-N)<1,
$$

which is equivalent to (15).

Remark. If $\sqrt{d}+P>2$, then by Theorem 2.3 and inequalities (7) condition (15) can hold only if $N / k$ is an even convergent of the simple continued fraction expansion of $\sqrt{d}-P$. If

$$
\sqrt{d}-P=\left[c_{0} ; \overline{c_{1}, \ldots, c_{m-1}, c_{m}}\right]
$$

then by (9) we have

$$
\frac{1}{\left(c_{2 n+1}+2\right) D_{2 n}^{2}}<(\sqrt{d}-P)-\frac{C_{2 n}}{D_{2 n}}<\frac{1}{c_{2 n+1} D_{2 n}^{2}}
$$

for all $n \in \mathbb{N}$. Thus, if there exists a $c_{2 n+1}>\sqrt{d}+P$, then by Theorem 5.3 expansion (14) is the least $\mathrm{dn}_{N}$ expansion of $\tau_{0}$ when $N=C_{2 n+l m}$ and $k=D_{2 n+l m}$ for any $l \in \mathbb{N}$. By contrast, if $c_{2 n+1}+2<\sqrt{d}+P$ for all $n$, then (14) is never the least $\operatorname{dn}_{N}$ expansion of $\tau_{0}$.

TheOREM 5.4. Let $\tau_{0}=\sqrt{d}$ where $d$ is a positive integer and not a perfect square. If $m$ is a positive integer, then

$$
\sqrt{d}=\left\langle 2 k d, 2\left(k^{2} d-m^{2}\right), \overline{k^{2} d-m^{2}}\right\rangle_{2 m},
$$

where $k$ is a positive integer such that $k \sqrt{d}>m$. Expansion (18) is the least $\mathrm{dn}_{2 m}$ expansion of $\sqrt{d}$ if and only if

$$
0<\sqrt{d}-\frac{m}{k}<\frac{1}{2 k \sqrt{d}}
$$

Proof. Let $k$ be a positive integer such that $k \sqrt{d}>m$. By choosing $a_{1}=2 k d$ we get from the $\operatorname{dn}_{N}$ algorithm

$$
\tau_{1}=\frac{2 k d}{\sqrt{d}}-2 m=2(k \sqrt{d}-m)>0 .
$$

We continue by choosing $a_{2}=2\left(k^{2} d-m^{2}\right)>0$ and get

$$
\tau_{2}=\frac{2\left(k^{2} d-m^{2}\right)}{2(k \sqrt{d}-m)}-2 m=k \sqrt{d}-m>0 .
$$

Finally, with $a_{3}=k^{2} d-m^{2}$ we have

$$
\tau_{3}=\frac{k^{2} d-m^{2}}{k \sqrt{d}-m}-2 m=k \sqrt{d}-m=\tau_{2}
$$


and hence we get periodic expansion (18).

Now $a_{1}=2 k d=\lceil 2 m \sqrt{d}\rceil$ if and only if $0<2 k d-2 m \sqrt{d}<1$ which is equivalent to (19). If inequality (19) holds, then

$$
0<a_{2}-2 m \tau_{1}=2(k \sqrt{d}-m)(k \sqrt{d}+m-2 m)<\frac{1}{2 d}
$$

and

$$
0<a_{3}-2 m \tau_{2}=(k \sqrt{d}-m)(k \sqrt{d}+m-2 m)<\frac{1}{4 d}
$$

so $a_{2}=\left\lceil 2 m \tau_{1}\right\rceil$ and $a_{3}=\left\lceil 2 m \tau_{2}\right\rceil$. Hence expansion (18) is the least $\mathrm{dn}_{2 m}$ expansion of $\sqrt{d}$ if and only if inequality (19) holds.

REMARK. By (17) inequality (19) has infinitely many solutions in $m / k$ for every $\sqrt{d}$, as we may choose $m=C_{2 n}$ and $k=D_{2 n}$ when $n$ is large enough. Consequently every irrational $\sqrt{d}$ has infinitely many periodic least $\mathrm{dn}_{N}$ expansions.

EXAmPle 4. Periodic least $\mathrm{dn}_{N}$ expansions given by Theorem 5.3:

\begin{tabular}{|c|c|c|}
\hline$\tau_{0}$ & $N$ & least $\operatorname{dn}_{N}$ expansion of $\tau_{0}$ \\
\hline$\sqrt{K^{2}+1}$ & $K$ & $\left\langle\overline{K^{2}+1,1}\right\rangle_{K}$ \\
$\sqrt{2}$ & 7 & $\langle 10, \overline{1,50}\rangle_{7}$ \\
$\frac{1+\sqrt{5}}{2}$ & 1 & $\langle 2, \overline{1,4}\rangle_{1}$ \\
$\frac{-2+\sqrt{13}}{3}$ & 5 & $\langle 3, \overline{4,9}\rangle_{5}$ \\
\hline
\end{tabular}

Periodic least $\mathrm{dn}_{N}$ expansions given by Theorem 5.4:

\begin{tabular}{|c|c|c|}
\hline$\tau_{0}$ & $N$ & least $\operatorname{dn}_{N}$ expansion of $\tau_{0}$ \\
\hline$\sqrt{2}$ & 14 & $\langle 20,2, \overline{1}\rangle_{14}$ \\
$\sqrt{3}$ & 10 & $\langle 18,4, \overline{2}\rangle_{10}$ \\
$\sqrt{6}$ & 44 & $\langle 108,4, \overline{2}\rangle_{44}$ \\
\hline
\end{tabular}

\begin{tabular}{|c|c|c|}
\hline$\tau_{0}$ & $N$ & least $\mathrm{dn}_{N}$ expansion of $\tau_{0}$ \\
\hline$\sqrt{7}$ & 13 & $\langle 35,3, \overline{2,59,2}\rangle_{13}$ \\
\hline $3+\sqrt{2}$ & 1 & $\langle 5,1,7, \overline{1,14}\rangle_{1}$ \\
\hline$\frac{6+\sqrt{3}}{2}$ & 5 & $\langle 20,1,4, \overline{1,2,2,1,5,5}\rangle_{5}$ \\
\hline
\end{tabular}

Other periodic least $\mathrm{dn}_{N}$ expansions:

\section{Bounded partial numerators}

One of the major open questions of Diophantine approximation is if the simple continued fraction expansions of algebraic numbers of degree greater than 2 have bounded partial denominators. In the case of $\mathrm{dn}_{N}$ expansions the analogue is quickly solved. In fact, we show below that for every positive 
real number there exists a $\mathrm{dn}_{N}$ expansion that has partial numerators from a set of two digits only.

Lemma 6.1. Let $\alpha_{1}$ and $\alpha_{2}$ be positive integers such that $\alpha_{1}<\alpha_{2}$ and

$$
\alpha_{1} \alpha_{2} /\left(\alpha_{1}+\alpha_{2}\right) \geq N^{2}
$$

and denote

$$
\begin{gathered}
\tau_{m}=\frac{-\left(N^{2}+\alpha_{2}-\alpha_{1}\right)+\sqrt{\left(N^{2}+\alpha_{2}-\alpha_{1}\right)^{2}+4 \alpha_{1} N^{2}}}{2 N}, \\
\tau_{M}=\frac{-\left(N^{2}-\alpha_{2}+\alpha_{1}\right)+\sqrt{\left(N^{2}+\alpha_{2}-\alpha_{1}\right)^{2}+4 \alpha_{1} N^{2}}}{2 N}=\tau_{m}+\frac{\alpha_{2}-\alpha_{1}}{N}
\end{gathered}
$$

and $I=\left[\tau_{m}, \tau_{M}\right]$. If $\tau_{0} \in I$, there exists a $\operatorname{dn}_{N}$ expansion $\tau_{0}=\left\langle a_{1}, a_{2}, \ldots\right\rangle_{N}$ such that $a_{i} \in\left\{\alpha_{1}, \alpha_{2}\right\}$ for all $i$.

Proof. As the positive solutions $x$ to equations

$$
x=\frac{\alpha_{1}}{N+\frac{\alpha_{2}}{N+x}} \text { and } x=\frac{\alpha_{2}}{N+\frac{\alpha_{1}}{N+x}}
$$

are $x=\tau_{m}$ and $x=\tau_{M}$, respectively, it follows that

$$
\tau_{m}=\left\langle\overline{\alpha_{1}, \alpha_{2}}\right\rangle_{N} \text { and } \tau_{M}=\left\langle\overline{\alpha_{2}, \alpha_{1}}\right\rangle_{N} .
$$

Let us denote $T_{1}(x)=\alpha_{1} /(N+x)$ and $T_{2}(x)=\alpha_{2} /(N+x)$ for $x \in I$. Then

$$
T_{1}\left(\tau_{M}\right)=\tau_{m}, \quad T_{2}\left(\tau_{m}\right)=\tau_{M}, \quad T_{1}\left(\tau_{m}\right)=\frac{\alpha_{1}}{\alpha_{2}} \tau_{M} \text { and } T_{2}\left(\tau_{M}\right)=\frac{\alpha_{2}}{\alpha_{1}} \tau_{m} .
$$

Because

$$
\begin{aligned}
& \frac{\alpha_{1}}{\alpha_{2}} \tau_{M}=\frac{\alpha_{1}}{\alpha_{2}}\left(\tau_{m}+\frac{\alpha_{2}-\alpha_{1}}{N}\right) \geq \frac{\alpha_{2}}{\alpha_{1}} \tau_{m} \\
& \Leftrightarrow \frac{\alpha_{1}\left(\alpha_{2}-\alpha_{1}\right)}{\alpha_{2} N} \cdot \frac{\alpha_{1} \alpha_{2}}{\alpha_{2}^{2}-\alpha_{1}^{2}}=\frac{\alpha_{1}^{2}}{N\left(\alpha_{2}+\alpha_{1}\right)} \geq \tau_{m} \\
& \Leftrightarrow \quad \frac{2 \alpha_{1}^{2}}{\left(\alpha_{2}+\alpha_{1}\right)}+N^{2}+\alpha_{2}-\alpha_{1} \geq \sqrt{\left(N^{2}+\alpha_{2}-\alpha_{1}\right)^{2}+4 \alpha_{1} N^{2}} \\
& \Leftrightarrow \quad \frac{\alpha_{1}^{3}}{\alpha_{2}+\alpha_{1}}+\alpha_{1}\left(N^{2}+\alpha_{2}-\alpha_{1}\right) \geq\left(\alpha_{2}+\alpha_{1}\right) N^{2} \\
& \Leftrightarrow \quad \frac{\alpha_{1} \alpha_{2}}{\alpha_{2}+\alpha_{1}} \geq N^{2},
\end{aligned}
$$

then inequality (20) implies that $T_{1}\left(\tau_{m}\right) \geq T_{2}\left(\tau_{M}\right)$. Therefore

$$
T_{1}(I) \cup T_{2}(I)=\left[\tau_{m}, T_{1}\left(\tau_{m}\right)\right] \cup\left[T_{2}\left(\tau_{M}\right), \tau_{M}\right]=\left[\tau_{m}, \tau_{M}\right]=I .
$$


Let $\tau_{0} \in I$. As the functions $T_{1}$ and $T_{2}$ are injective on $I$, then by (21) there exists a $\tau_{1} \in I$ such that

$$
\tau_{0}=\frac{a_{1}}{N+\tau_{1}} \quad \Leftrightarrow \quad \tau_{1}=\frac{a_{1}}{\tau_{0}}-N,
$$

where $a_{1} \in\left\{\alpha_{1}, \alpha_{2}\right\}$. Similarily, if $\tau_{i} \in I$, then there exists a $\tau_{i+1} \in I$ such that

$$
\tau_{i}=\frac{a_{i+1}}{N+\tau_{i+1}} \Leftrightarrow \tau_{i+1}=\frac{a_{i+1}}{\tau_{i}}-N
$$

where $a_{i+1} \in\left\{\alpha_{1}, \alpha_{2}\right\}$. It follows by induction that $\tau_{0}=\left\langle a_{1}, a_{2}, a_{3}, \ldots\right\rangle_{N}$, where $a_{i} \in\left\{\alpha_{1}, \alpha_{2}\right\}$ for all $i$.

TheOREM 6.2. Let $\tau_{0}$ be a positive real number. Then there exist positive integers $\alpha_{1}$ and $\alpha_{2}$ such that $\tau_{0}=\left\langle a_{1}, a_{2}, \ldots\right\rangle_{N}$, where $a_{i} \in\left\{\alpha_{1}, \alpha_{2}\right\}$ for all $i$.

Proof. Due to Lemma 6.1 it is sufficient to show that there exist positive integers $\alpha_{1}$ and $\alpha_{2}$ such that $\alpha_{1}<\alpha_{2}, \alpha_{1} \alpha_{2} /\left(\alpha_{1}+\alpha_{2}\right) \geq N^{2}$ and $\tau_{0} \in\left[\tau_{m}, \tau_{M}\right]$, where $\tau_{m}$ and $\tau_{M}$ are as in Lemma 6.1. Now

$$
\begin{aligned}
\tau_{m} & =\frac{-\left(N^{2}+\alpha_{2}-\alpha_{1}\right)+\sqrt{\left(N^{2}+\alpha_{2}-\alpha_{1}\right)^{2}+4 \alpha_{1} N^{2}}}{2 N} \\
& =\frac{N^{2}+\alpha_{2}-\alpha_{1}}{2 N}\left(-1+\sqrt{1+\frac{4 \alpha_{1} N^{2}}{\left(N^{2}+\alpha_{2}-\alpha_{1}\right)^{2}}}\right) .
\end{aligned}
$$

Since the function

$$
f(x)=x\left(-1+\sqrt{1+\frac{\alpha_{1}}{x^{2}}}\right)
$$

is strictly decreasing and tends to 0 as $x$ tends to infinity for all positive $\alpha_{1}$, then

$$
\tau_{m}<\tau_{0}<\tau_{m}+\frac{\alpha_{2}-\alpha_{1}}{N}=\tau_{M}
$$

when $\alpha_{2}-\alpha_{1}$ is large enough. Because

$$
\frac{\alpha_{1} \alpha_{2}}{\alpha_{1}+\alpha_{2}}=\frac{1}{1 / \alpha_{1}+1 / \alpha_{2}} \geq \frac{\alpha_{1}}{2},
$$

then we may choose $\alpha_{1} \geq 2 N^{2}$ and $\alpha_{2}$ such that $\alpha_{2}-\alpha_{1}$ is large enough for (22) to hold true.

EXAMPLE 5. Here are the first 20 digits of some $\mathrm{dn}_{1}$ expansions with bounded numerators.

\begin{tabular}{|c|c|c|}
\hline$\tau_{0}$ & $\{a, b\}$ & bounded $\operatorname{dn}_{N}$ expansion of $\tau_{0}$ \\
\hline$\sqrt[3]{2}$ & $\{2,4\}$ & $\langle 2,2,4,2,4,2,2,2,2,2,4,4,4,4,4,4,4,2,2,4, \ldots\rangle_{1}$ \\
$\pi$ & $\{2,5\}$ & $\langle 5,2,5,2,2,5,5,2,2,2,2,5,5,2,2,5,2,5,5,5, \ldots\rangle_{1}$ \\
$e$ & $\{3,7\}$ & $\langle 7,3,3,7,7,7,3,3,7,3,7,3,3,7,3,3,3,7,3,3, \ldots\rangle_{1}$ \\
$\ln 2$ & $\{2,4\}$ & $\langle 2,4,2,2,4,4,4,4,4,4,4,4,2,2,4,2,2,4,4,4, \ldots\rangle_{1}$ \\
\hline
\end{tabular}


Acknowledgements The author would like to thank Jaroslav Hančl, Tapani Matala-aho and Kalle Leppälä for their helpful comments.

\section{References}

[1] Anselm M., Weintraub S.H., A generalization of continued fractions, J. Number Theory 131 (2011), 2442-2460

[2] Berndt B.C., Ramanujans Notebooks, Part II, Springer-Verlag, New York, USA, 1989

[3] Berndt B.C., Ramanujans Notebooks, Part III, Springer-Verlag, New York, USA, 1991

[4] Borwein J., Crandall R., Fee G., On the Ramanujan AGM fraction. Part I: the realparameter case, Exp. Math. 13 (2004), 275-286

[5] Borwein J., van der Poorten A., Shallit J., Zudilin W., Neverending Fractions: An Introduction to Continued Fractions, Austral. Math. Soc. Lect. Ser. 23, Cambridge University Press, UK, 2014

[6] Dutka J., Wallis's product, Brouncker's continued fraction, and Leibniz's series, Arch. History Exact Sci. 26 (1982), 115-126.

[7] Lorentzen L., Waadeland H., Continued Fractions with Applications, Stud. Comput. Math. 3, North-Holland Publishing Co., Amsterdam, 1992

[8] Rosen K. H., Elementary Number Theory and its applications, 3rd edition, AddisonWesley Publishing Company, USA, 1992

Research unit of mathematical sciences

P.O. Box 8000, 90014 University of Oulu, Finland 